\title{
Front-loaded (FL) microscopic approach against Conventional approach for detecting Acid Fast Bacilli (AFB) in sputum
}

\author{
Mohammad Murad Hossain, ${ }^{1}$ Md. Robed Amin, ${ }^{1}$ Ahmedul Kabir, ${ }^{2}$ Md. Zobaer Ahmed, ${ }^{3}$ Lilian Catherene Gomes, ${ }^{4}$ \\ Shehreen Ahmed, ${ }^{5}$ Md. Torikul Islam ${ }^{6}$
}

\begin{abstract}
Introduction: Tuberculosis (TB) is one of the world's deadliest disease. Presently, one fourth of the world's population is thought to be infected with TB. Early diagnosis of tuberculosis is very important for therapeutic reasons and to control the spread of infection. The purpose of this study was to compare the efficacy of fluorescence (FL) microscopy in comparison to Ziehl- Neelsen (ZN) staining

Methods: A cross-sectional study of 310 TB suspects attending Dhaka Medical College hospital subjected to four sputum examinations by ZN and FM. Löwenstein- Jensen culture was used as the gold standard. Of 310 suspects enrolled, 304 (98\%) had a complete diagnostic work-up involving three sputum specimens for ZN and FM, culture and chest $X$-ray (CXR).

Result: Out of 320 suspected case, 10 persons were out of our study. Among 310, 204 suspected case sputum culture was positive. Though we took sputum culture as gold standard, so there were 204 tuberculosis patient present in our study. Out of 204 TB patient I25 (6I.2\%) cases were positive in ZN staining. Negative ZN methods were also high 79 (39.7\%) which indicate less sensitivity.

On the other hand, FM methods showed 193 (94.6\%) cases positive out of 204 sputum culture positive patient. It also presence of II cases which showed negative result in this methods. From this data we can assumed higher sensitivity of FL methods from ZN methods.

Here 106 cases were culture negative. Out of these 106 people only 05 persons were positive in ZN staining whereas 10 persons were find to positive in FL methods. This message demonstrates us that specificity of $Z N$ methods are slightly higher over FM methods.

Overall sensitivity of ZN microscopic (6I\%)) methods are much lower than FL microscopic methods (95\%). On the other hand, specificity was slightly reverse, which showed $95.5 \%$ in case ZN methods and $91 \%$ in FL methods.

It also demonstrates that positive predictive value of ZN microscopy (96\%) is slightly higher than $\mathrm{FL}$ microscopic (95\%) methods but negative predictive value showed gross difference ZN microscopic (56\%) and FL microscopic (89\%) methods.

Conclusions: The FL methods is more sensitive and shortens the diagnostic process of tuberculosis. Consequently, more patients can be put on a regimen for smear-positive TB, contributing to improved treatment and reducing transmission. For that Front-loaded (FL) microscopic approach may apply for early diagnosis and detection of tuberculosis.
\end{abstract}

Key words: tuberculosis; diagnosis; Ziehl-Neelsen; fluorescence microscopy.

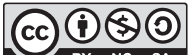

DOI: https://doi.org/l0.3329/jom.v2lil.44107

Copyright: (C) 2020 Khanom M. This is an open access article published under the Creative Commons Attribution-NonCommercial-NoDerivatives 4.0 International License, which permits use, distribution and reproduction in any medium, provided the original work is properly cited, is not changed in any way and it is not used for commercial purposes.

Received: 25 July, 2019;

Accepted: 14 September, 2019

1. Associate Professor of Medicine, Dhaka Medical College

2. Professor of Medicine, Dhaka Medical College

3. Indoor Medical Officer (Medicine), Dhaka Medical College

4. Medical Officer, Department of Physical Medicine \& Rehabilitation, BSMMU

5. Lecturer, Department of Pharmacology, Dhaka Medical College

6. Registrar (Neurology), Dhaka National Medical College

Corresponding author: Dr. Md. Robed Amin, Associate Professor of Medicine, Dhaka Medical College. Email: robedamin@yahoo.com.

\section{Introduction:}

Tuberculosis (TB) is an infectious disease that can be deadly if not treated properly. In 2014, 9.6 million people were infected with TB worldwide and 1.5 million died from the disease. Over $95 \%$ of mortality related to TB occur in low- and middleincome countries. The South-East Asia region accounts for a disproportionately high number of global TB cases and Bangladesh is one of 22 'high TB-burden' countries. According to the WHO annual estimated incidence for all 
cases is 225 per 100000 populations per year. The prevalence is estimated to be 434 per 100000 populations. In 2014, there were 187,005 new cases of TB in Bangladesh and it was the leading cause of death. ${ }^{1}$

TB can occur any age group, but older persons are more vulnerable. It is the world's greatest infectious killer of reproductive aged women $\&$ the leading cause of death among people with HIV/AIDS. ${ }^{2}$

At first Robert Koch identified \& described the tubercle bacilli as causative organism of tuberculosis on March 24th, $1882 \&$ he received the Nobel prize for his discovery. This acid fast bacillus can cause pulmonary as well as extrapulmonary tuberculosis like meningeal Tuberculosis, pleural TB, pericardial TB, Bone TB etc. ${ }^{3}$

A complete medical evaluation for Tuberculosis must include a medical history, physical examination, chest X-ray and microbiological examination (sputum or some other appropriate sample). It may also include a tuberculin skin test, other scan and X-ray and surgical biopsy.

Direct sputum smear microscopy by conventional light microscope using Zeil-Neelsen (ZN) stain is the most widely used test option of TB diagnosis in most of the hospitals. But it has some disadvantages like poor sensitivity and labor intensiveness. The standard method requires three sputum specimens to be obtained over 2 days as on the spot, morning and another on the spot. Many studies have shown that third smear does not add too much to the overall case finding rate. Yassin \& Cuevas (2003) in their study reported that third sputum sample only identifies from $1 \%$ to $5 \%$ of patients who could be missed by 1 st and 2 nd sputum sample. ${ }^{4} \mathrm{Wu}$ et al. (2000) and Kivihya-Ndugga et al (2003) also reported similar finding in two different studies. ${ }^{5,6}$ A systematic review in 2007 recommended the reduction of the number of sputum examinations from three to two for the betterment of global TB control programme. ${ }^{7}$ Upon that review WHO recently has changed their policy and reduced the minimum number of sputum specimen examinations from three to two. ${ }^{8}$ But the issue of timing of collection of sputum has not been specifically addressed. So patient still needs two days to complete diagnosis. The method of sputum collection can be front-loaded (FL) by collecting an additional sputum one hour after 1st sample. The FL staining is regarded as a more sensitive \& reliable method for detection of tubercle bacilli as FL stain can detect bacilli when they are present around 104/ml of sputum, while Z-N stain requires 105 bacilli/ml. There is a stronger binding of mycolic acid of bacilli with phenol auramine. For that FL staining is more sensitive. It is better screening test \& bacilli can be seen in low power objective. ${ }^{9,10}$ Ramsay et al (2009) recently published a large series over 923 patients conducted in Nigeria, Ethiopia,
Yemen and Nepal and compare the FL approach (spot-Xspot) with conventional approach (spot-morning) and got good results. ${ }^{11}$ In this study the FL approach (spot-Aspot) was compared with conventional approach (spot-morning) using ZN stain. Spot-Aspot-morning scheme was also being compared with spot-morning - 2nd spot scheme. Culture of the sputum was done as gold standard.

\section{Material and Methods:}

This cross sectional study was conducted at the department of Medicine, Dhaka Medical college hospital during the period of June 2018 to January 2019. 320 Study participant were selected on the basis of defined selection criteria following the purposive sampling methods, where inclusion criteria were clinically suspected cases sent to TB center, patients whose age 18-75 years, cough for e" 3 weeks. Written inform consent was taken before the study. A semi structured questionnaire was developed to collect the data. After collection, it was analyzed using SPSS version 20 and result were tabulated.

\section{Work Plan/Study procedure:}

Four (4) sputum samples was collected. First spot sample on first visit followed by another extra sample one hour after that which was labeled as A-spot. Next day morning sample and another spot $\left(4^{\text {th }}\right)$ sample was collected. Smears from each sample was stained using ZN stain and read by light microscope. The slides were graded according to WHO and the International Union Against Tuberculosis and Lung Disease (IUATLD) standard operational guideline in to five categories such as negative, scanty,,+++ and +++ . The yield of spot-Aspot scheme was compared with the yield of spot-morning scheme and the yield of Front-loaded spotAspot-morning scheme with the yield of spot-morning-2 ${ }^{\text {nd }}$ spot scheme. Sensitivity and specificity were determined against Culture (gold standard).

\section{Result:}

A total of 320 suspects were enrolled in the study. Among them, 4 suspected cases third sputum culture result was missing, making it difficult to establish the final diagnosis. Another 6 suspects had no CXR results, mainly because they did not return to collect their sputum results, which are necessary to establish a diagnosis of smear-negative TB. For $310(97 \%)$ suspects the data set was complete, and included results on three specimens per suspect for ZN, FL and culture, together with a CXR reading.

Among the sample, Male participant were 191 (61.6\%) and female were 119 (38.4\%). The male:female ratio among the suspects was 1.6. Majority participant 121 (39.1\%) are belonging to more than 55 years of age. Male were $72(37.7 \%)$ 
and female were $49(41.2 \%)$. From 18 to 35 years of age male were $51(26.7 \%)$ and female were $31(26.1 \%)$. Age in between 36 to 54 years male patients were $68(35.6 \%)$ and female were $37(31.1 \%)$ (Table 1$)$.

Table 1: Distribution of patient according to age

\begin{tabular}{lccc}
\hline Age group & Male & Female & Total (\%) \\
\hline $18-35$ & 51 & 31 & $26.5 \%$ \\
$36-54$ & 68 & 37 & $33.9 \%$ \\
$>55$ & 72 & 49 & $39.1 \%$ \\
\hline Total & 191 & 119 & \\
\hline
\end{tabular}

Of the 310 TB suspects, 204 (66\%) were culture positive. Among the culture positive patient $\mathrm{ZN}$ microscopy shows positive of 125 (61.2\%), whether FL microscopic method shows 193 (94.6\%) positivity. Great amount 79 (38.7\%) of participant had false negative in $\mathrm{ZN}$ methods. On the other hand, sputum negative sample was 106 (34.1\%). Here ZN microscopic methods showed 05 (4.71\%) positive but FL methods showed $10(9.4 \%)$ positivity (Table-2).

Table 2: Test characteristics for Ziehl-Neelsen (ZN) and fluorescence microscopy (FM) stratified by sample collection using culture as gold standard

\begin{tabular}{lccccc}
\hline Specimen & Sputum & $\begin{array}{c}\text { ZN } \\
(+v e)\end{array}$ & $\begin{array}{c}\text { ZN } \\
(-v e)\end{array}$ & $\begin{array}{c}\text { FL } \\
(+v e)\end{array}$ & $\begin{array}{c}\text { FL } \\
(-v e)\end{array}$ \\
\hline Culture (+ve) & 204 & 125 & 79 & 193 & 11 \\
& & $(61.2 \%)$ & $(38.7 \%)$ & $(94.6 \%)$ & $(5.3 \%)$ \\
Culture (-ve) & 106 & $\begin{array}{c}05 \\
(4.7 \%)\end{array}$ & $\begin{array}{c}101 \\
(96.2 \%)\end{array}$ & $\begin{array}{c}10 \\
(9.4 \%)\end{array}$ & $\begin{array}{c}96 \\
(90.5 \%)\end{array}$ \\
\hline
\end{tabular}

Among all the 310 patients these two methods are applied and showed significant result. We also determine sensitivity, specificity, positive predictive value and negative predictive value. (Figure 1). Sensitivity is very high in FL methods comparison to $\mathrm{ZN}$ method, but specificity is slightly low. But both positive and negative predictive value of FL methods are very high. Here we can see sensitivity of $\mathrm{ZN}$ microscopic methods was $61 \%$ but in FL methods it was $95 \%$. Specificities of ZN methods $95.5 \%$ but FL methods was $91 \%$. Positive predictive value and negative predictive value of $\mathrm{ZN}$ methods was $96 \%$ and $56 \%$. But in case of FL methods it was $95 \%$ and $89 \%$.

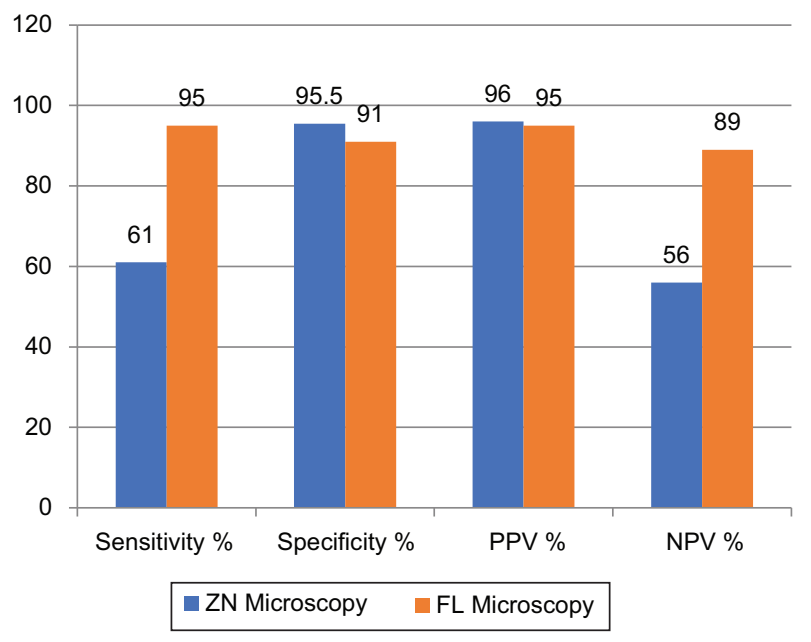

Figure 1: Bar chart shows sensitivity, specificity, PPV and NPV of the ZN and FL methods.

\section{Discussion:}

In the present study we compared results of light microscopy of Z-N stain smear with that of Front loaded (FL) microscopy for detection of AFB.

Here we see the majority of the patient (39.1\%) are over the age of 55 years. It noticed that old aged person is more prone to developed tuberculosis rather than younger and middle aged person. From this value we can suspect that immunosuppression can be a major factor for developing tuberculosis. Relation of older age and immunosuppression are very closed. Older person is more vulnerable to Diabetes Mellitus, Chronic Kidney diseases, Chronic liver diseases. These are major cause of immunosuppression thus provoked many infectious diseases like tuberculosis.

There is another message that is female participants are fewer then male. (Male- 191, Female- 119). It signifies that females were less oriented about this study due to their lack of knowledge. It also showed their less interest due to their social and familial restriction. In our society majority women are neglected to their diseases. Same type of study showed in (K. deshai et al 2009) ${ }^{17}$.

Out of 320 suspected case, 10 persons were out of our study. Among 310, 204 suspected case sputum culture was positive. Though we took sputum culture as gold standard, so there were 204 tuberculosis patients present in our study. Out of 204 TB patient $125(61.2 \%)$ cases were positive in $\mathrm{ZN}$ staining. Negative ZN methods also high 79 (39.7\%). Which indicate less sensitivity.

On the other hand, FL methods showed 193 (94.6\%) cases positive out of 204 sputum culture positive patient. It also 
presence of 11 cases which showed negative result in this methods. From this data we can assume higher sensitivity of FL methods from ZN methods.

In this study we can see 106 cases were culture negative. This showed that 106 persons are TB free. Out of these 106 people only 05 persons were positive in ZN staining whereas 10 persons were found positive in FL methods. This message demonstrates that specificity of $\mathrm{ZN}$ methods are slightly higher over FM methods.

Overall sensitivity of ZN microscopic (61\%)) methods are much lower than FL microscopic methods (95\%). On the other hand, specificity was slightly reverse, which showed 95.5\% in case ZN methods and 91\% in FL methods.

In case of positive predictive value ZN microscopy (96\%) showed slightly higher than FL microscopic (95\%) methods but negative predictive value showed gross difference $\mathrm{ZN}$ microscopic (56\%) and FL microscopic (89\%) methods.

Same results were obtained by Githui et al ${ }^{12}$ ( $80 \%$ by fluorescent microscopy $\& 65 \%$ by ZN staining), Ulukanligil et $\mathrm{al}^{13}$ also obtained $85.2 \%$ positivity by fluorescent microscopy \& $67.6 \%$ by ZN method. Similar results obtained by S J Murray et al ${ }^{14}$ ( $93 \%$ by Fl microscopy $\& 73 \%$ by Z-N method), Bavaikar JN et a a $^{15}(69 \%$ by F1 microscopy \& 50\% by Z-N method) and A. Jain et $\mathrm{al}^{16}$ ( $41 \%$ by Fl microscopy \& $32 \%$ by Z-N method).

This results shows that FL method is more sensitive as compared to $\mathrm{ZN}$ staining method for demonstration of AFB. This may be because organisms in FL methods offer much contrast, appearing as brilliant-yellow against a more or less darkened background. As low power is used for examination, large number of specimens can be examined in a given time and colour-blind investigators may also use this method without difficulties these are an additional advantages of FL microscopy.

From this study it is clear that FL is a more sensitive technique than $\mathrm{ZN}$ methods.

In conclusion, FL microscopic methods is more appropriate in detecting AFB from pulmonary samples then $\mathrm{ZN}$ stain. This methods time is also saved. For that in high workload area it is more suitable screening method. On the other hand, sensitivity, specificity also carry a good result.

So for screening of pulmonary Tuberculosis Front-loaded (FL) microscopic approach is very much effective than other conventional methods.

\section{Acknowledgments:}

We thank the Director, Dhaka Medical College Hospital and the staff of the Medicine ward. We are also thankful to DOTS center, Department of Microbiology and Radiology imaging of Dhaka Medical College.

Conflict of interest: The Bangladesh Medical Research Council funded this study.

\section{References:}

1. World Health Organization.WORLD TB DAY 2016: Bangladesh continues its battle against the disease. (Online) (Cited 2018 august 04). Available from URL: http:// www.searo.who.int/bangladesh/world-tb-day-2016/en/

2. K Prasanthi, AR Kumari (2005). "Efficacy of fluorochrome stain in the diagnosis of pulmonary tuberculosis co- infected with HIV". Indian Journal of Medical Microbiology.;23 (3):179-185

3. Vasantha Kumari R (1995). "Sputum smear examination. A view point". Indian Journal of Tuberculosis;42:135-7.

4. Yassin, M.A \& Cuevas, L.E. (2003) "How many sputum smears are necessary for case finding in pulmonary tuberculosis?" Trop Med Int Health, 8 (10), pp.927-32.

5. Wu, Z.L \& Wanq, A.Q. (2000) “Diagnostic yield of repeated smear microscopy examinations among patients suspected of pulmonary TB in Shandong province of China". International Journal of Tuberculous lung Disease, 4 (11), pp. 1086-7.

6. Kivihya-Ndugga, L.E, Van-cleeff, M, R Githui, W, A et al. (2003) “A comprehensive comparison of Ziehl-Neelsen and fluorescence microscopy for the diagnosis of tuberculosis in a resource poor urban setting". International Journal of Tuberculous lung Disease 7 (12), pp. 1163-71.

7. Mase, S, R, Ramsay, A, Vivien, N et al. (2007) "Yield of serial sputum specimen examinations in the diagnosis of pulmonary tuberculosis: a systematic review". International Journal of Tuberculous lung Disease, 11 (5), pp.485-95.

8. World Health Organization 2007, New WHO policies, viewed May 10 2009, http://www.who.int/tb/dots/ laboratory/policy/en/index.html.

9. Betty A Forbes, Daniel F Sahm, Alice S. Weissfield, Bailey \& Scott's Diagnostic Microbiology. 10th edn. Mosby publishers;1998. p. 715-43.

10. Satya Sri S. Text book of Pulmonary and Extra pulmonary Tuberculosis. 4th edition. Inter Print Publishers; 2000. p. 63-9.

11. Ramsay, A, Yassin, M.A, Cambanis, A et al. (2009) FrontLoading Sputum Microscopy Services An Opportunity to Optimise Smear-Based Case Detection of Tuberculosis in High Prevalence Countries. Journal of Tropical Medicine Article ID 398767,Vol- 2009 pp. 1-6 
12. Githui W, Kitui F, Juma ES, Obwana DO, Mwai J, Kwamasga D. (1993) "A comparative study on the reliability of the fluorescence microscopy \& Ziehl-Neelsen method in the diagnosis of pulmonary tuberculosis". East African Medical Journal .;70:263-6.

13. Ulukanligil M, Aslan G, Tasci S (2000). “A comparative study on the different staining methods and number of specimens for the detection of acid-fast bacilli". Memórias do Instituto Oswaldo Cruz.;95:855-8.

14. S J Murray, A Barrett, JG Magee, R Freeman (2003). "Optimization of acid fast smears for the direct detection of mycobacteria in clinical samples". Journal of Clinical Pathology:;56;613-615.
15. Banvaliker JN, Gupta R, Sharma DC, Goel MK, Kumari S (1997). "HIV seropositivity in hospitalized pulmonary tubercilosis patients in Delhi”. Indian Journal of Tuberculosis; 44:17-9.

16. A Jain, A Bhargawa, SK Agarwal. (2002) "A comparative study of two commonly used staining techniques for acid fast bacilli in clinical specimens". Indian Journal of Tuberculosis.;49;161.

17. K. deshai, S. malek, C. mehtaliya, (2009) Comparative study of $\mathrm{ZN}$ staining $\mathrm{V} / \mathrm{S}$ flurochrome stain from pulmonary and extra pulmonary tuberculosis, Gujrat medical journal., 64 (2): Page-32-34. 